\title{
Haematocervix after Conization Diagnosed by
} Ultrasonography

\begin{tabular}{|l|l|l|}
\hline H. & Helmut & Pschera \\
\hline A. & Anders & Kjaeldgaard \\
\hline
\end{tabular}

Department of Obstetrics and Gynecology, Huddinge University Hospital, Huddinge, Sweden

\section{Key Words}

Haematocervix

Conization

Ultrasonography

Abstract

Haematocervix is an uncommon complication after conization. This report deals with 1 case that presented with clinical signs of progressive stenosis and was diagnosed by ultrasonography. Based on analysis of the 3 previously reported cases the pathogenesis of this condition is discussed.

Helmut Pschera, MD, Department of Obstetrics and Gynecology, Huddinge University Hospital, S-141 86 Huddinge (Sweden)

Introduction

Stenosis of the cervical canal after conization is a well-known late postoperative complication, and haema-tometra can be demonstrated in about $1 \%$ of the patients undergoing cold knife cone biopsy [1]. However, postoperative occlusion of the cervix may also result in haematocervix. This rare condition is characterized by enlargement of the cervix which is dilated by accumulated menstrual blood. In this report we present 1 case, diagnosed by ultrasonography, and discuss the pathogenesis of this complication.

Case Report

A 30-year-old woman (gravida 3, para 3) was referred to our department because of severe cervical intraepithelial neoplasia (CIN 3) demonstrated in biopsies from endocervix and ectocervix. Cold knife cone cervical biopsy was performed and 4 polyglycolic acid (Dexon $\left.{ }^{\circledR}\right)$ sutures were applied to the cervix to secure haemo-stasis. A primary haemorrhage at $10 \mathrm{~h}$ postoperatively was controlled by applying two supplementary sutures to the cervix under general anaesthesia, and the patency of the cervical canal was controlled by passing an uterine sound into the cervical canal. Tranex-amic acid was given during hospitalization, and the patient was discharged after 4 days. Histological examination of the resected cervical tissue measuring 8 $\times 2 \times 0.5 \mathrm{~cm}$ showed CIN 3 with clear resection margins.

At routine follow-up 3.5 months later the patient presented with amenorrhoea for 7 weeks. After the operation she had 3 periods which were quite normal concerning interval (24-26 days), duration (5-6 days) and menstrual blood loss. However, during the 3rd 


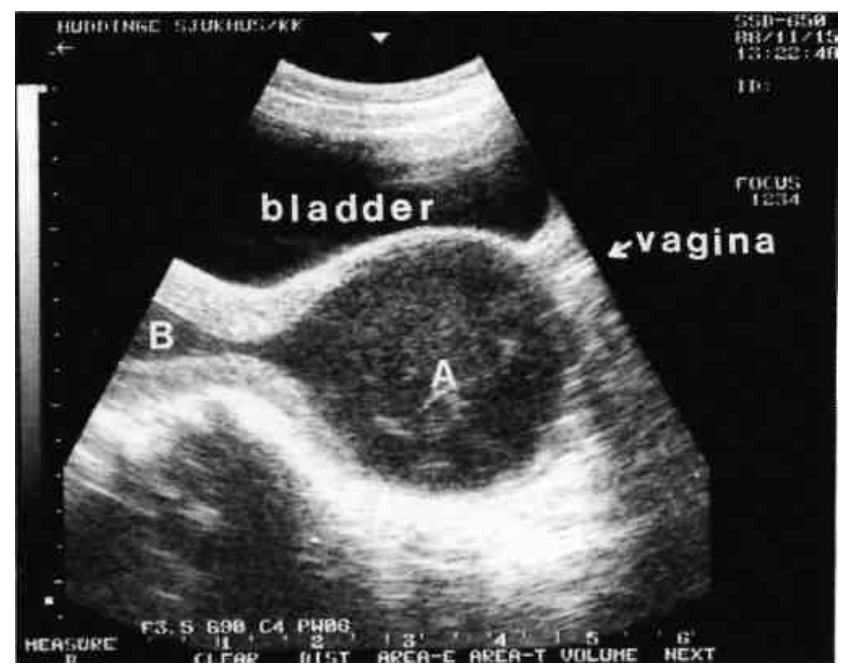

Fig. 1. Longitudinal sonographic scan showing haematocervix (A) and slightly enlarged uterine cavity (B).

period she experienced dysmenorrhoea, and during the amenorrhoea period she complained of severe dysmenorrhoea-type pains at the time of the two expected periods. On gynaecological examination the external cervical os could not be identified, and the cervix was replaced by an approximately $8 \mathrm{~cm}$ large cystic mass, on the top of which a slightly enlarged uterus was palpated. A pregnancy test was negative. Pelvic ultrasonography demonstrated an enlarged cervix measuring $8 \times 8 \times 8 \mathrm{~cm}$. The dilated cervical canal contained fluid of low echodensity and was surrounded by a thin cervical wall. The uterine cavity was slightly enlarged (fig. 1). These findings, in conjunction with the history, established the diagnosis of haematocervix combined with a moderate degree of haematometra.

310

Pschera/Kj aeldgaard

Under general anaesthesia, an incision of the scar in the top of the vagina indicating the site of the occluded external os was performed, and $300 \mathrm{ml}$ of chocolate-coloured blood was obtained. A size $12 \mathrm{~mm}$ drain was inserted intracervically and left for 6 days. Then a copper IUD was inserted in order to prevent reocclusion of the cervical canal and external os. The uterine sound passed $11 \mathrm{~cm}$. No antibiotic prophylactic was given, and the postoperative course was uneventful. Reexamination after 3 and 7 weeks, respectively, showed anatomical normalization on gynaecological and ultrasound examination. At the last follow-up visit 10 months after the reoper-ation the patient was still well with normal periods.

Discussion

The literature offers no explanation of the pathogene-sis of postoperative haematocervix. The 3 previously reported cases all presented with a history of absent menstrual bleeding postoperatively [2, 3]. However, in the present case the haematocervix developed in spite of 3 normal menstrual periods following the conization. This indicates a progressive stenosis and finally total obliteration of the external cervical os without a concomitant stenosis of the cervical canal. We suggest that the distal part of the cervix reduced by conization may represent a locus minoris resistentiae which can be more easily dilated by the accumulated menstrual blood than the thicker and more rigid wall surrounding the uterine cavity. Consequently, a slight 
enlargement of the uterine cavity as found in the present case should be expected and was also demonstrated in the previously reported cases [2, 3].

In the present case the diagnosis of haematocervix was not suspected before the ultrasound examination. However, the awareness of this rare complication may

spare the patient unnecessary abdominal surgery as illustrated by the 2 cases which were not diagnosed until peroperatively in connection with hysterectomy [2]. Since haematocervix can be successfully treated by incision of the stricture obliterating the external os, the main clinical problem is the correct diagnosis. In this respect, ultrasound examination offers a valuable diagnostic tool as evident from the present case. Ultrasonography is also a reliable technique for demonstrating haematometra and should therefore be considered in all cases of amen-orrhoea after cone biopsy.

References

Luesley, D.M.; McCrum, A.; Terry, P.B.; Wade-Evans, T.; Nicholson, H.O.; Mylotte, M.J.; Emens, J.M.; Jordan J.A.: Complications of cone biopsy related to the dimensions of the cone and the influence of prior colposcopic assessment. Br. J. Obstet. Gynaec. 92: 158-164(1985). Clark, A.; McKay Hart, D.; McCune, G.; Cordiner, J.: Haematocervix following cone biopsy of the cervix. J. Obstet. Gynaec. Br. Commonw. 80: 858-861 (1973).

Konstantoulas, M.: Ultrasonic diagnosis of haematocervix following cone biopsy of the cervix. Br. J. Radiol. 51: 56-57 (1978). 